\title{
Identificacion del agente causal del mildiu de la albahaca y metodos para la estimacion de su severidad
}

\author{
Identification of basil downy mildew and severity estimation
}

\author{
Alejandro Risco Mendoza ${ }^{1 *}$, Walter Eduardo Apaza Tapia ${ }^{2}$ y Luz Leonor Mattos Calderon ${ }^{3}$ \\ * Autor de correspondencia
}

\begin{abstract}
Resumen
Esta investigación tuvo como objetivo la identificación del agente causal del mildiú de la albahaca y la validación de un método de estimación de la severidad. El patógeno presenta morfométricamente zoosporangióforos monopodiales y ramificados dicotómicamente en ángulo agudo hasta 4,25 veces en la parte terminal, un largo de 320,1 $\mu \mathrm{m}$; esterigmas cortos de $15,5 \mu \mathrm{m}$ y de $17,9 \mu \mathrm{m}$ de largo. Los zoosporangios, ligeramente ovoides con 27,7 $\mu \mathrm{m}$ de diámetro y con 31,9 $\mu \mathrm{m}$ de largo. La estimación de severidad se realizó por el método de Horsfall y Barratt (HB) (Horsfall y Barratt, 1945), por estimación visual directa (VD) y evaluando daño foliar por cada tercio de la planta (TH). La evaluación aleatoria se realizó en tres oportunidades por tres evaluadores, y los valores estimados fueron correlacionados con la severidad real. Para medir la confiabilidad entre evaluadores y métodos de evaluación se empleó el coeficiente de correlación y concordancia (CCC). En el método VD, los tres evaluadores obtuvieron valores de 0,57; 0,63 y 0,44 respectivamente; en el método de HB fueron 0,$58 ; 0,46$ y 0,42 y en TH 0,$14 ; 0,08$ y 0,52 . Se concluye que, el agente causal del mildiú de la albahaca es Peronospora belbahrii y según el CCC el mejor método de evaluación fue el de la estimación visual directa.
\end{abstract}

Palabras clave: mildiú albahaca; severidad estimada; severidad real; coeficiente de correlación; Peronospora belbahrii; Ocimum basilicum.

\begin{abstract}
This research is aimed to identify the causal agent of basil downy mildew and the validation of a method for estimating severity. The pathogen shows morphometrically, monopodial and dichotomically zoosporangiophores branched in acute angle up to 4.25 times at the upper ends, with a length of $320.12 \mu \mathrm{m}$; the shorter branchlets are $15.5 \mu \mathrm{m}$ and the longer are $17.9 \mu \mathrm{m}$. The zoosporangia slightly ovoid with $27.7 \mu \mathrm{m}$ in diameter and $31.9 \mu \mathrm{m}$ in length. The evaluation of severity was carried out by the Horsfall and Barratt (HB) method (Horsfall and Barratt, 1945), by direct visual estimation (VD) and by evaluating leaf damage for each third of the plant (TH). The randomized evaluation was carried out three times, with the participation of three raters. Each of them estimated the severity independently; the values obtained were correlated with the real severity, to obtain reliability between evaluators and evaluation methods, the correlation and concordance coefficient (CCC) was applied. In the VD method the three raters obtained values of $0.57 ; 0.63$ and 0.44 respectively; in the HB method, the values of $0.58 ; 0.46$ and 0.42 and in $\mathrm{TH} 0.14 ; 0.08$ and 0.52 . According to the morphometric data, the causal agent of basil downy mildew is Peronospora belbahrii and according to CCC the best method of evaluation was the direct visual estimation.
\end{abstract}

Keywords: basil downy mildew; estimated severity; actual severity; correlation coefficient; Peronospora belbahrii; Ocimum basilicum.

\section{Introducción}

La albahaca (Ocimum basilicum Lin.) es una planta aromática y medicinal, originaria de Asia Meridional y ampliamente distribuida en regiones tropicales y subtropicales (Makri \& Kintzios, 2008). La producción nacional de la albahaca en el Perú va en incremento, desde 2539 toneladas métricas en el 2008 hasta 6687 toneladas métricas en el año 2014 (INEI, 2015). Estos incrementos pueden significar que a mayor área sembrada también las epidemias se intensifiquen, siempre que las condiciones ambientales sean favorables para su desarrollo, principalmente aquellas que afectan a las hojas que es la parte de la planta que se comercializa.

Entre las enfermedades foliares de la albahaca se encuentra el mildiú, considerada y registrada como la más importante en otros países del mundo. Esta enfermedad como tal, ha sido descrita entre 19 géneros de patógenos, como agentes causales en diversos cultivos, caso Peronospora, Pseudoperonospora, Basidiophora, Benua, Bremia, Novotelnova, Parapenospora, Plasmopara, 
Plasmoverna, Protobremia, Hyaloperonospora, Perofascia, Graminivora, Poakatesthia, Viennotia entre otros (Thines et al., 2009). Siendo Peronospora belbahrii el agente causal del mildiú de la albahaca que fue reportado por primera vez por Belbahrii et al. (2005) y anteriormente considerada como Peronospora lammi. Este patógeno, taxonómicamente pertenece al reino Chromista, división Oomycota, clase Oomycetes, orden Peronosporales y familia Peronosporaceae (Corda, 1837).

En el Perú, no hay reportes de enfermedades foliares para el cultivo de la albahaca, sin embargo, en diversos países del mundo existe información sobre $P$. belbahrii donde indican que es el agente causal del mildiú de la albahaca (Thines et al., 2009), así lo demuestran los primeros reportes sobre este patógeno, en diversos países como República Checa (Šafránková \& Holková, 2014 y Petrželová et al., 2015), Brasil (Saude et al., 2013), Korea (Choi et al., 2016), China (Kong et al., 2015), entre otros. Los investigadores señalan que no solo afecta a albahaca, sino también, a otros cultivos como Coelus (Rivera et al., 2016).

El mildiú es considerado un parásito biótrofo y policíclico, por lo que, su manejo puede ser a través del uso de variedades tolerantes y aplicación de productos químicos. Además, se conoce su capacidad de desarrollar resistencia a fungicidas, que es otro problema que se tiene que considerar en su manejo. Collina et al. (2016) y Cohen et al. (2013), hacen referencia que $P$. belbahrii tiene resistencia a Mefenoxam, fungicida ampliamente usado para el control de esta enfermedad.

En el Perú, no existen reportes sobre métodos de evaluación estandarizados para monitorear al mildiú de la albahaca. En otras condiciones a la nuestra, cada investigador evalúa esta enfermedad según sus propios objetivos o criterios. Homa et al. (2014) midieron la severidad para encontrar el mejor control de fungicidas, utilizando una escala ordinal jerárquica que va de 0 a 3. El autor consideró, $0=$ ausencia de esporulación, $1=$ ligera esporulación, $2=$ esporulación moderada y $3=$ mucha esporulación en el envés de las hojas. Por otra parte, Danielsen y Ames (2008), plantearon la evaluación del mildiú de la quinua tomando como referencia la severidad relativa de tres hojas, una de cada tercio de la planta, las que se promedian para considerar la severidad de una planta completa. Mientras que, Horsfall y Barratt (1945), propone otra escala muy conocida, que actualmente se emplea para evaluar enfermedades de plantas y consta de 12 clases, cada una con rangos de porcentajes de severidad y el punto medio del rango.

Los sistemas o metodologías de evaluación son muy relativos y dependerán del objetivo del investigador. La estimación de la severidad de las enfermedades en los cultivos, es sumamente útil y necesaria para cuantificar el resultado de muchos estudios, pero principalmente para medir la gravedad de la enfermedad y poderla controlar.

Por lo expuesto, esta investigación tuvo como objetivos la identificación del agente causal del mildiú de la albahaca y la validación de un método para la estimación de la severidad de esta enfermedad, causado por P. belbahrii.

\section{Materiales y métodos \\ Condiciones de campo y toma de muestras}

Entre los meses de abril a junio del 2016, con temperaturas entre 15 y $25^{\circ} \mathrm{C}$ y humedad relativa entre 65 y 90 por ciento, fueron colectadas de los campos del Programa de Hortalizas de la Universidad Nacional Agraria La Molina (UNALM), 100 ramas de albahaca (Ocimum basilicum L.) de la variedad del Ligure con síntomas típicos de mildiú. De la muestra tomada, se seleccionaron 30 ramas, teniendo en consideración el número de hojas, porte erecto, ausencia de ramas laterales, longitud aproximada de $30 \mathrm{~cm}$ y presencia de síntomas de mildiú en diferentes grados de severidad. Estas fueron llevadas a un ambiente con luz para poder visualizar los síntomas al momento de la evaluación; fueron colocadas en recipientes de vidrio con agua, para mantener la turgencia y evitar que se marchiten en todo el proceso de evaluación. Estos recipientes, con sus respectivas ramas, fueron etiquetadas con códigos alfanuméricos para que los evaluadores no puedan recordar en cada repetición.

\section{Identificación morfológica}

Se efectuaron preparaciones en porta objetos, a partir las hojas con síntomas cloróticos en cuyo envés presentaban material polvoso que consistía en las estructuras del patógeno. Estas estructuras fueron visualizadas en el microscopio a un aumento de $400 \mathrm{X}$, fueron fotografiadas y medidas en micras $(\mu)$ para su identificación. En la identificación morfológica se emplearon claves de diferentes autores. Clements y Shear (1931) para identificar a nivel de género y tomando como referencia de Thines et al. (2009) para la identificación a nivel de especie. Adicionalmente se emplearon otras referencias para corroborar los datos.

\section{Prueba de patogenicidad}

Se sembraron semillas de albahaca del cultivar Sel Ligure en bandejas con sustrato Sun Shine Nro. 3. Al cabo de dos semanas los plantines fueron trasplantados en macetas contenidas con sustrato estéril que consistió en, tierra de cultivo, arena fina de río y compost en proporción de 1:1:1. Las plantas se dejaron crecer hasta que desarrollaron seis hojas en promedio, esto ocurrió a los 14 días después del trasplante. Para la obtención de inóculo, se colectaron hojas de albahaca mostrando en el haz áreas cloróticas y en el envés la presencia de esporulación plomiza. Las hojas colectadas fueron lavadas a presión con agua destilada estéril, especialmente sobre las zonas con esporulación, a fin de remover los esporangios del patógeno y poder colectarlos, para ello se usó un vaso de precipitación. A la suspensión obtenida (aproximadamente $1 \times 10^{8} / \mathrm{mL}$ ) se le adicionó un adherente siliconado (Break Thru) a dosis de $0,5 \mathrm{cc} /$ litro. La suspensión preparada fue asperjada, usando un pulverizador, tanto en el haz como el envés de 
las hojas y se realizó en horas de la tarde (5:00 p.m.). Cada macetero, con su respectiva planta inoculada, fue colocado dentro de una bolsa de polietileno transparente, con un filtro de algodón en el extremo superior durante doce días $y$, luego hacer la evaluación de la presencia o ausencia de la enfermedad para poder cumplir con los postulados de Koch.

\section{Determinación de la severidad estimada (SE)}

La evaluación de severidad, se realizó en las ramas seleccionadas con aproximadamente $30 \mathrm{~cm}$ de longitud que fueron consideradas como unidad muestral. Estas ramas colocadas en recipientes de vidrio codificados presentaron hojas enfermas en diferentes grados de severidad y estuvieron en un ambiente iluminado y separadas a cierta distancia, unas de otras, para facilitar su evaluación.

La evaluación de la severidad del mildiú, de las treinta muestras codificadas, se realizó en tres oportunidades por tres evaluadores (Eval ${ }_{1}, E_{2} a_{2}$ y Eval ${ }_{3}$ en forma aleatoria, cada uno independientemente para determinar la severidad estimada (SE) y expresada en porcentajes. Para este fin, se emplearon tres metodologías de evaluación: i) Escala de Horsfall y Barratt (HB) (Horsfall y Barratt, 1945), ii) escala de Danielsen y Ames (2008), que fue propuesta para evaluar mildiú de la quinua, en la que proponen evaluar tres hojas (TH), una de cada tercio, y estos tres valores promediados para representar la severidad de la enfermedad en la planta completa, iii) estimación visual directa de la planta completa (VD). La escala de Horsfall y Barratt (HB) cuenta con 12 clases: Clase $1=$ severidad de $0 \%=$ punto medio $0 \%$; Clase $2=$ severidad de 0 a $3 \%=$ punto medio $1,5 \%$; Clase $3=$ severidad de 3 a $6 \%=$ punto medio 4,5 $\%$; Clase $4=$ severidad de 6 a $12 \%=$ punto medio $9 \%$; Clase $5=$ severidad de 12 a $25 \%=$ punto medio $18,5 \%$; Clase $6=$ severidad de 25 a $50 \%=$ punto medio $37,5 \%$; Clase $7=$ severidad de 50 a $75 \%=$ punto medio $62,5 \%$; Clase $8=$ severidad de 75 a $87 \%=$ punto medio $81 \%$; Clase $9=$ severidad de 87 a $94 \%=$ punto medio $90,5 \%$; Clase $10=$ severidad de 94 a $97 \%=$ punto medio $95,5 \%$; Clase $11=$ severidad de 97 a $100 \%=$ punto medio 98,5 $\%$; Clase $12=$ severidad del $100 \%=$ punto medio 100 $\%$. La segunda propuesta, por Danielsen y Ames (2008), consta de una escala diagramática, numérica y jerárquica con valores discretos de $0 \%, 1,5 \%, 10 \%, 20 \%, 30 \%, 40$ $\%, 50 \%, 60 \%, 70 \%, 80 \%, 90 \%, 95 \%$ y $100 \%$. Finalmente la propuesta de esta investigación es por estimación visual directa (VD) donde se consideró a la planta completa como unidad muestral.

\section{Determinación de la severidad real (SR)}

Para calcular el valor de la SR de cada unidad muestral (planta completa) se empleó el método modificado de Nita et al. (2003), donde todas las plantas fueron defoliadas por completo, se hicieron recortes de las áreas cloróticas de cada hoja, obteniendo el peso de las áreas cloróticas y el peso total de cada hoja. Por diferencia de pesos en gramos, se calculó la severidad real en porcentaje a través de la siguiente fórmula.

$\mathrm{SR}=(\mathrm{PL} / \mathrm{PT}) \times 100$

Donde, SR. severidad real; $P L$. Peso se las lesiones; $P T$. Peso total.

\section{Validación de la escala}

Los valores de la SR (x) del mildiú de la albahaca, fueron correlacionados con los valores de la SE (y) obtenidos a través de las tres metodologías de evaluación (TH, HB y VD). Para validar las escalas de evaluación se empleó el Coeficiente de Correlación y Concordancia de Lin $\left(\mathrm{P}_{\mathrm{c}} \mathrm{o}\right.$ $\mathrm{CCC}_{\mathrm{Lin}}$ ) o índice de reproducibilidad (Lin, 1989), cuyo resultado se obtuvo con el producto del coeficiente de correlación $(r)$ y el sesgo o coeficiente que mide el error $\left(\mathrm{C}_{\mathrm{b}}\right)$. Para ello además, se requirió el uso de las desviaciones estándares de los valores medios o estimados (y) y de los valores reales o verdaderos $(\mathrm{x})$.

$\mathrm{CCC}_{\text {lin }}=\mathrm{r}_{\mathrm{b}} \mathrm{C}_{\mathrm{b}}$

Donde,

$$
\mathrm{C}_{b}=\frac{2}{V+\frac{1}{V}+u^{2}}, \quad \mathrm{u}=\frac{\left(u_{y}+u_{x}\right)}{\sqrt{\left(\sigma_{y} * \sigma_{x}\right)}}, \quad \mathrm{v}=\frac{\sigma_{y}}{\sigma_{x}}
$$

\section{Resultados y discusión}

\section{Resultados}

\section{Síntomas y observación morfológica}

Los síntomas observados en los campos afectados, fueron típicos a los que muestran los mildiús en otros cultivos; en el haz, los síntomas se iniciaron en las hojas basales observandose clorosis internerval y conforme la enfermedad avanzaba, la clorosis era más pronunciada hasta que finalmente se producía la defoliación; mientras que, en el envés de las áreas cloróticas se observó el signo, que consistió en esporulación de color plomizo con aspecto polvoso (Figura 1).

En el presente trabajo de investigación, las dimensiones de las estructuras estuvieron dentro de los rangos determinados por Clements y Shear (1931). El patógeno presentó morfométricamente zoosporangioforos monopodiales y ramificados dicotómicamente en ángulo agudo hasta 4,25 veces en la parte terminal, con un largo de 320,1 $\mu \mathrm{m}$; dos tipos de esterigmas curvados, uno largo y el otro corto. Los esterigmas cortos con aproximadamente $15,5 \mu \mathrm{m}$ y los largos con 17,9 $\mu \mathrm{m}$. Los zoosporangios de forma ligeramente ovoide con base redondeada, de color entre marrón oscuro y oliváceo, con longitud promedio de $31,9 \mu \mathrm{m}$ y diámetro promedio de $27,7 \mu \mathrm{m}$ y con $1,2 \mathrm{de}$ relación de longitud y diámetro del zoosporangio (Figura 2 y Tabla 1). 


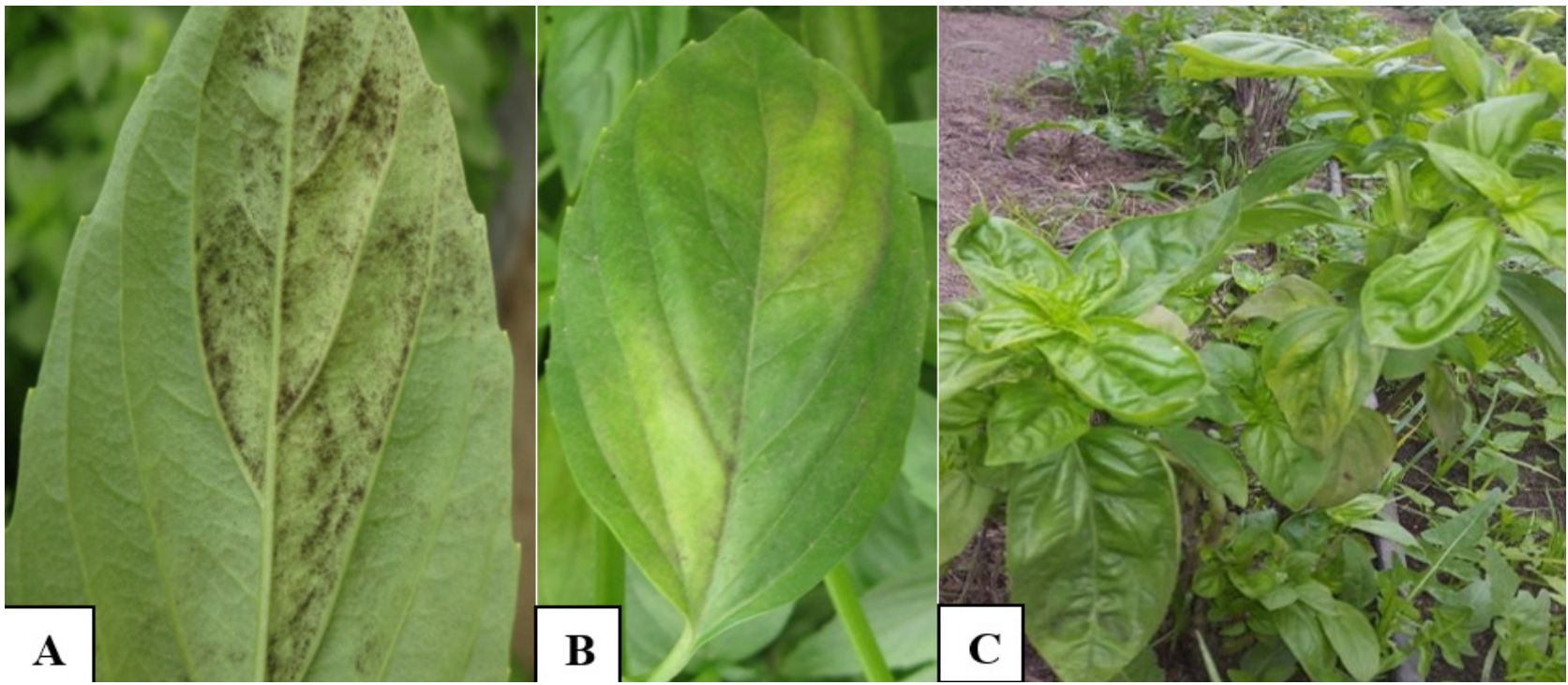

Figura 1. Plantas en campo. A. Síntomas de clorosis en el haz de las hojas basales de planta de albahaca B. Síntomas avanzados en hojas, C. Signo de esporulación plomiza en el envés de la hoja
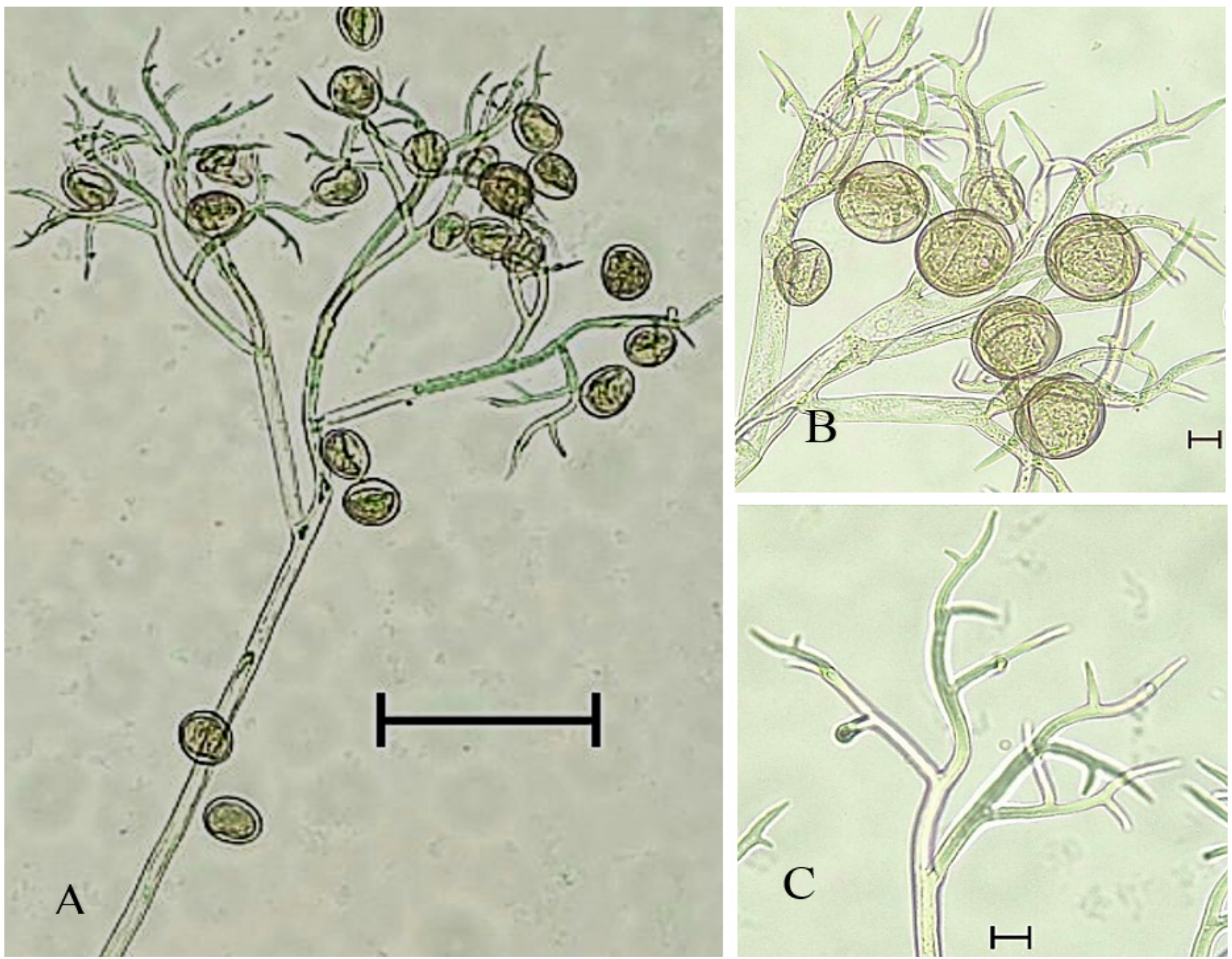

Figura 2. Estructuras asexuales de $P$. belbahii. A. Zoosporangióforo monopodial, arborescente ramificado dicotómicamente con zoosporangios globosos ligeramente ovoides; B. Zoosporangios; C. Esterigmas largos y cortos. Barra $(\mathrm{A})=100 \mu \mathrm{m}$; barra $(\mathrm{B}$ y $\mathrm{C})=10 \mu \mathrm{m}$ 
Tabla 1. Características morfométricas de $P$. belbahrii causante de la enfermedad del mildiú de la albahaca. Universidad Nacional Agraria La Molina - Lima - Perú, 2017

\begin{tabular}{|c|c|c|c|c|c|}
\hline \multirow{2}{*}{$\begin{array}{c}\text { Esporangióforo } \\
(\mu \mathrm{m})\end{array}$} & \multirow{2}{*}{$\begin{array}{l}\text { Ramificación } \\
\left(\mathrm{N}^{\circ}\right)\end{array}$} & $\begin{array}{l}\text { Esterigma } \\
(\mu \mathrm{m})\end{array}$ & $\begin{array}{r}\text { Zoospor } \\
(\mu \mathrm{m}\end{array}$ & $\begin{array}{l}\text { rangio } \\
\text { a) }\end{array}$ & \multirow{2}{*}{$\begin{array}{c}\text { Relación } \\
\text { L/D del } \\
\text { zoosporangio }\end{array}$} \\
\hline & & Largo Corto & $\begin{array}{l}\text { Diámetro } \\
\text { (D) }\end{array}$ & $\begin{array}{l}\text { Largo } \\
(\mathrm{L})\end{array}$ & \\
\hline 320,1 & 4,3 & $17,9 \quad 13,5$ & 27,7 & 32,0 & 1,2 \\
\hline
\end{tabular}

\section{Prueba de patogenicidad}

A los siete días después de la inoculación, las plantas mostraron síntomas iniciales muy semejantes a los que se observaron en campo. Estos consistieron en áreas cloróticas en las hojas y como signo esporulación plomiza con aspecto polvoso (Figura 3). A los doce días, el 100 por ciento de las hojas en cada planta, mostraron el signo de la enfermedad en distintos grados, similar a lo encontrado por Šafránková y Holková (2014).
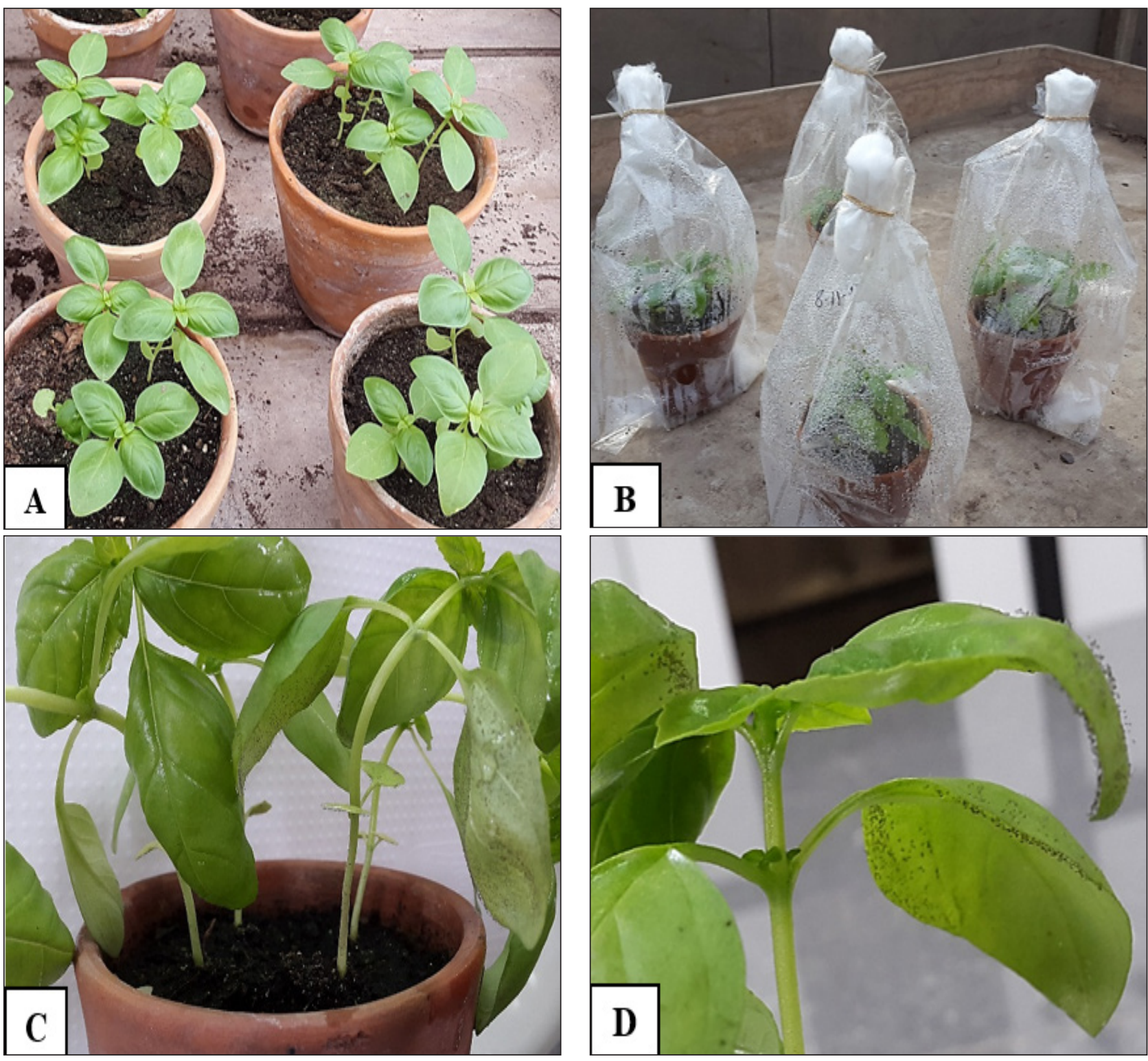

Figura 3. Prueba de patogenicidad. A. Plantas sanas; B. Plantas inoculadas, C. Plantas con síntomas a los 12 días después de la inoculación.; D. Presencia de signo sobre el haz y envés de las hojas

\section{Exactitud y precisión}

La correlación entre la SE (y) la SR (x) del mildiú mediante la metodología de tres hojas (TH) y el comparativo de la línea de tendencia de las evaluaciones con la línea de concordancia o línea perfecta se muestra en la fig. 4 y tabla 2. Al comparar la línea de concordancia con la línea de tendencia de los valores estimados de los tres evaluadores (Eval ${ }_{1}$, Eval $_{2}$ y Eval $_{3}$ ), se observa que hay una tendencia a sobrestimar cuando la severidad es menor a 30 por ciento y sobre este valor, hay una tendencia a subestimar. Los valores del coeficiente de correlación ( $r$ ) que es un índice de reproducibilidad fueron 0,$26 ; 0,17 \mathrm{y}$ 0,52 para cada evaluador respectivamente; los valores del Coeficiente de Correlación y Concordancia (CCC) que nos mide la exactitud y la precisión al mismo tiempo, fueron 0,$14 ; 0,08$ y 0,52 respectivamente. 

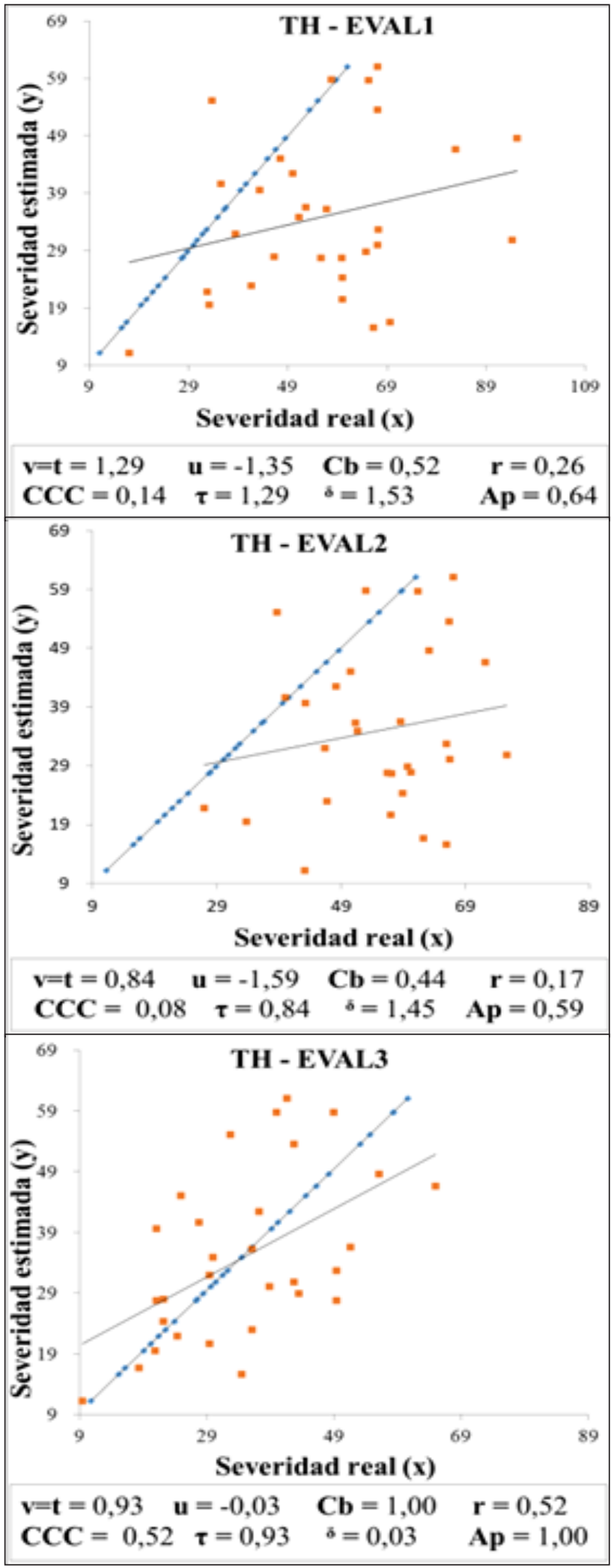

Figura 4. Estimación de la severidad del mildiú de la albahaca ( $P$. belbahrii) basado en la evaluación de tres hojas (TH) versus la severidad real por tres evaluadores $\left(\mathrm{Eval}_{1}, \mathrm{Eval}_{2} \mathrm{y} \mathrm{Eval}_{3}\right)$

Las líneas punteadas corresponden a la línea de concordancia, la línea sólida corresponde a la línea de mejor ajuste. $\mathbf{C}_{\mathrm{b}}$. Coeficiente que mide el error; r. coeficiente de correlación; CCC. Coeficiente de correlación y concordancia; Ap. Coeficiente concordancia mejorada.
La correlación entre la SE (y) y la SR (x) del mildiú, mediante la metodología de Horsfall y Barratt (1943), la línea de tendencia de las evaluaciones con la línea de concordancia o línea perfecta se muestra en la Figura 5 y Tabla 2. Al comparar la línea de concordancia con la línea de tendencia de los valores estimados de los tres evaluadores (Eval, $\mathrm{Eval}_{2}$ y $\mathrm{Eval}_{3}$ ), como se observa en la Figura 5, el Eval tiende a sobrestimar cuando los porcentajes son menores a 30 por ciento de severidad y cuando son superiores a este valor, el evaluador tiende a subestimar; sin embargo, el Eval y el Eval tienden a sobrestimar en todo momento. Los valores del coeficiente de correlación $(r)$ que es un índice de reproducibilidad fueron de 0,$62 ; 0,65$ y 0,70 para cada evaluador; el coeficiente de correlación y concordancia (CCC) que mide la exactitud y precisión, tuvo los valores de 0,$59 ; 0,46 \mathrm{y}$ 0,42 respectivamente.

La correlación entre la SE (y) y la SR (x) mediante la metodología de estimación visual directa (VD) y el comparativo entre la línea de tendencia de las evaluaciones con la línea de concordancia o línea perfecta, se muestra en la Figura 6 y Tabla 2. Al comparar la línea de concordancia con la línea de tendencia de los valores estimados de los tres evaluadores, el Eval ${ }_{1}$ y el Eval ${ }_{2}$ tienden a sobrestimar cuando los porcentajes son menores a 40 por ciento de severidad y cuando son superiores a este valor, los evaluadores subestiman; por su parte el Eval ${ }_{3}$ sobrestima en todo momento. Los valores del coeficiente de correlación (r) para cada evaluador fueron 0,$63 ; 0,65$ y $0,67 \mathrm{y}$ del Coeficiente de Correlación y Concordancia (CCC) fueron de 0,$58 ; 0,63$ y 0,44 respectivamente.

En la Tabla 2 se muestran los valores del coeficiente de correlación y concordancia (CCC) mediante la escala

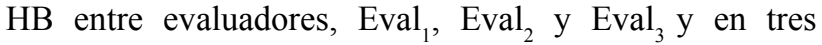
repeticiones o momentos de evaluación. En la primera repetición, los valores del CCC de los tres evaluadores son poco variables, 0,$50 ; 0,47$ y 0,57 respectivamente; en la segunda fueron 0,$47 ; 0,26$ y 0,34 , y en la tercera repetición 0,$51 ; 0,43$ y 0,28 . En estas dos últimas hay mayor variación respecto a la primera repetición; sin embargo, estos índices son superiores a los obtenidos mediante la escala TH que fue 0,$13 ; 0,10$ y 0,53 en la primera evaluación; 0,$15 ; 0,16$ y 0,47 en la segunda y 0,$12 ; 0,12$ y 0,37 y en la tercera. Con la propuesta de evaluación mediante la estimación visual directa (VD), los valores del CCC en la primera repetición fueron 0,$49 ; 0,46$ y 0,38 ; en la segunda 0,$47 ; 0,59$ y 0,40 y en la tercera 0,$53 ; 0,52$ y 0,45 respectivamente para los tres evaluadores. Como se puede evidenciar, los valores del $\mathrm{CCC}$ entre evaluadores y entre evaluaciones mediante la escala HB y TH tuvieron mayor variación con respecto a la escala VD, lo que indica que, en la propuesta VD se obtuvo mayor exactitud y mayor precisión, esto es corroborado con los datos promedios de este índice que fueron de 0,24 para TH, 0,49 para HB y 0,55 para VD.

\section{Discusión}

En diversos países del mundo se reportó a $P$. belbahrii 

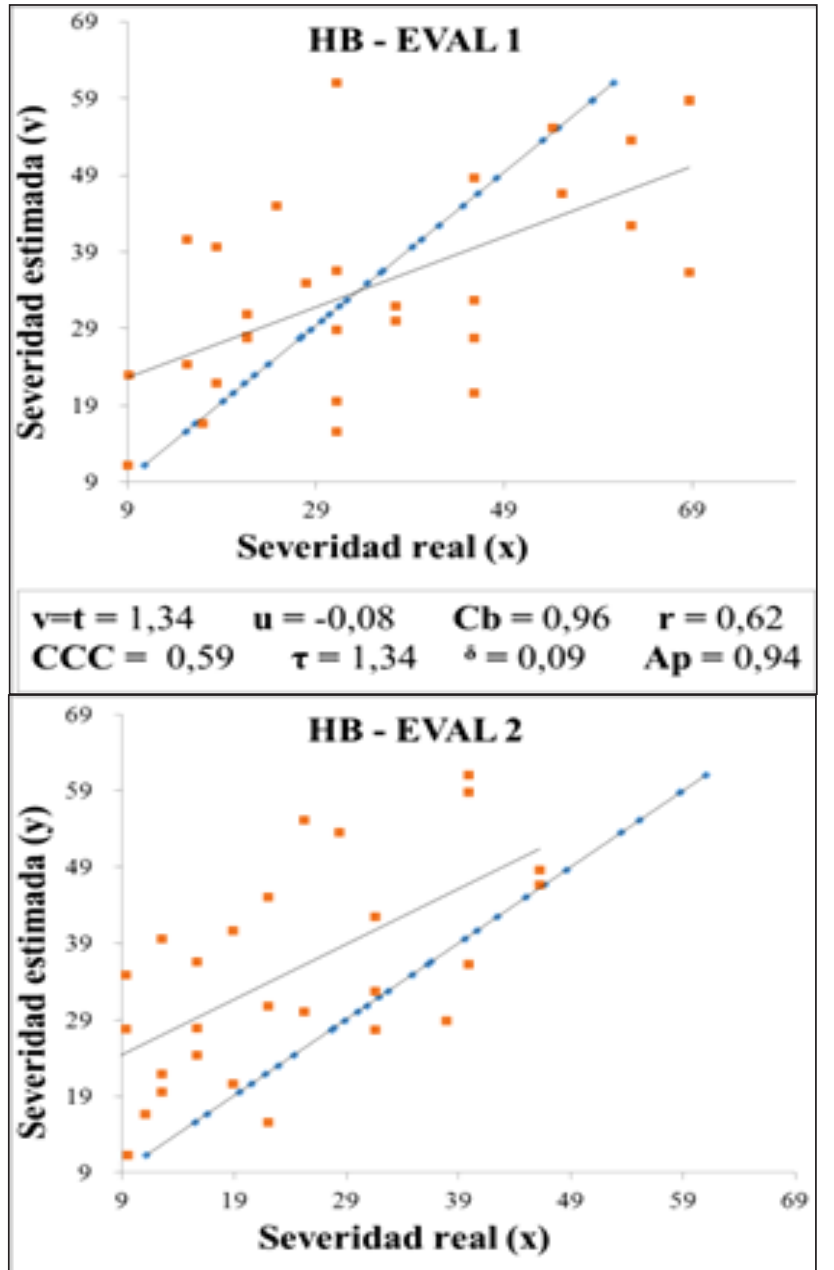

$\begin{array}{lccc}\mathbf{v}=\mathbf{t}=0,89 & \mathbf{u}=0,87 & \mathbf{C b}=0,72 & \mathbf{r}=0,65 \\ \mathbf{C C C}=0,46 & \boldsymbol{\tau}=0,89 & \delta=-0,82 & \mathbf{A p}=0,77\end{array}$

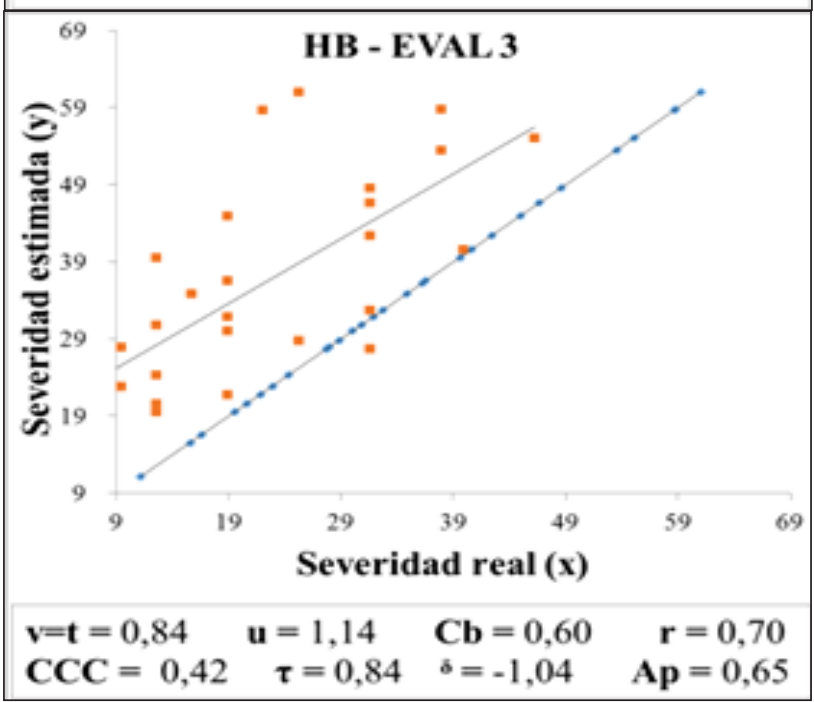

Figura 5. Estimación de la severidad del mildiú de la albahaca (P. belbahrii) basado en la escala de Horsfall y Barratt $(\mathrm{TH})$ versus la severidad real por tres evaluadores $\left(\right.$ Eval $_{1}$, Eval $_{2}$ y Eval $\left.{ }_{3}\right)$

La línea punteadas corresponden a la línea de concordancia, la línea sólida corresponde a la línea de mejor ajuste. $\mathbf{C}$. Coeficiente que mide el error; r. coeficiente de correlación; CCC. Coeficiente de correlación y concordancia; Ap. Coeficiente concordancia mejorada.

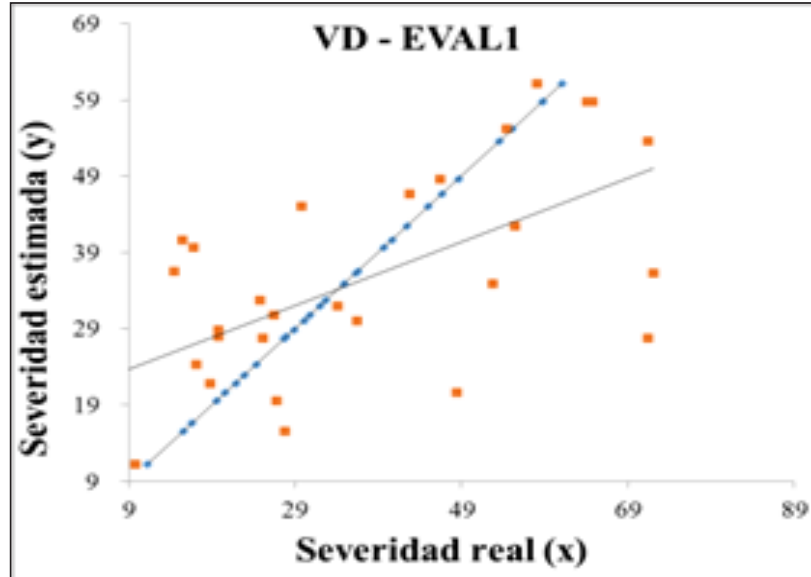

$\begin{array}{llll}\mathbf{v}=\mathbf{t}=1,51 & \mathbf{u}=-0,06 & \mathbf{C b}=0,92 & \mathbf{r}=0,63 \\ \mathbf{C C C}=0,58 & \boldsymbol{\tau}=1,51 & \delta=0,08 & \mathbf{A p}=0,88\end{array}$

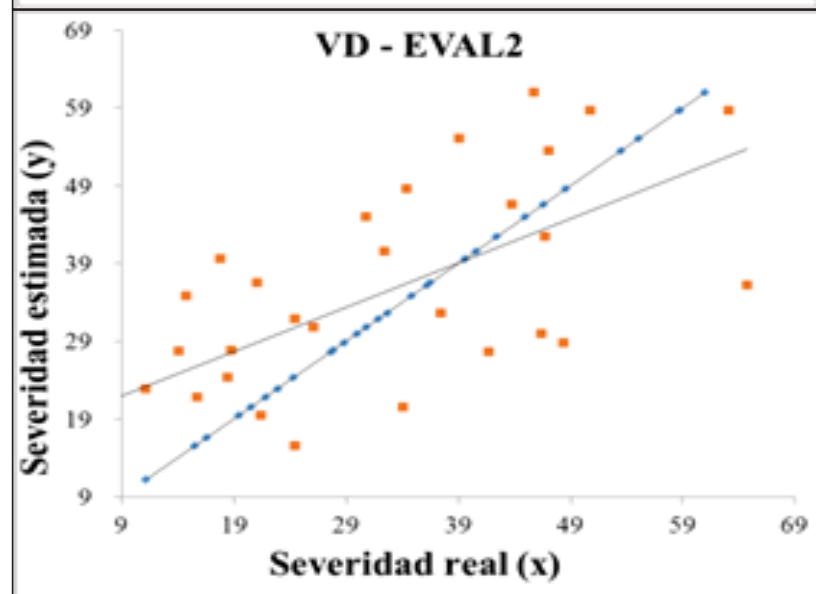

$\begin{array}{lccc}\mathbf{v}=\mathbf{t}=1,14 & \mathbf{u}=0,22 & \mathbf{C b}=0,97 & \mathbf{r}=0,65 \\ \mathbf{C C C}=0,63 & \boldsymbol{\tau}=1,14 & \delta \boldsymbol{b}=-0,23 & \mathbf{A p}=0,97\end{array}$

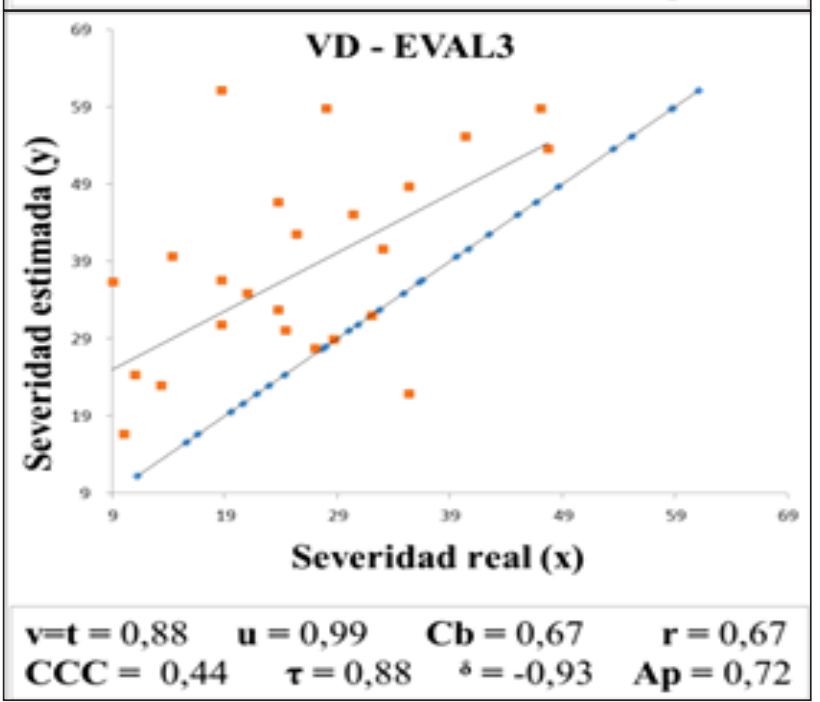

Figura 6. Estimación de la severidad del mildiú de la albahaca ( $P$. belbahrii) basado en estimación visual directa (VD) versus la severidad real por tres evaluadores $\left(\right.$ Eval $_{1}$, $\mathrm{Eval}_{2}$ y $\mathrm{Eval}_{3}$ ).

La línea punteadas corresponden a la línea de concordancia, la línea sólida corresponde a la línea de mejor ajuste. C. Coeficiente que mide el error; r. coeficiente de correlación; CCC. Coeficiente de correlación y concordancia; Ap. Coeficiente concordancia mejorada. 
Tabla 2. Coeficiente de correlación y concordancia (CCC), de la estimación de la severidad del mildiú de la albahaca causado por $P$. belbahrii, con tres métodos de evaluación (HB, VD y TH), tres repeticiones y tres evaluadores

\begin{tabular}{|c|c|c|c|c|c|c|c|c|c|c|c|c|}
\hline \multirow{3}{*}{ Evaluador } & \multicolumn{9}{|c|}{ Repetición } & \multirow{2}{*}{\multicolumn{3}{|c|}{ Promedio* }} \\
\hline & \multicolumn{3}{|c|}{$\mathrm{I}$} & \multicolumn{3}{|c|}{ II } & \multicolumn{3}{|c|}{ III } & & & \\
\hline & HB & VD & $\mathrm{TH}$ & $\mathrm{HB}$ & VD & $\mathrm{TH}$ & HB & VD & $\mathrm{TH}$ & $\mathrm{HB}$ & VD & $\mathrm{TH}$ \\
\hline EVAL 1 & 0,50 & 0,49 & 0,13 & 0,47 & 0,47 & 0,15 & 0,51 & 0,53 & 0,12 & 0.59 & 0.58 & 0.14 \\
\hline EVAL 2 & 0,47 & 0,46 & 0,10 & 0,26 & 0,59 & 0,16 & 0,43 & 0,52 & 0,12 & 0.47 & 0.63 & 0.08 \\
\hline EVAL 3 & 0,57 & 0,38 & 0,53 & 0,34 & 0,40 & 0,47 & 0,28 & 0,45 & 0,37 & 0.42 & 0.44 & 0.52 \\
\hline
\end{tabular}

*valores calculados con promedios de severidad de cada metodología. Promedio Total de CCC: VD. 0,55; HB. 0,49; TH. 0,24

como agente causal del mildiú de la albahaca (Saude et al., 2013; Šafránková \& Holková, 2014, Petrželová et al., 2015, Kong et al., 2015, Choi et al., 2016). Sin embargo, en el Perú en las zonas de La Molina, Huaral, Cañete, entre otros, se ha observado una enfermedad en el cultivo de albahaca que tiene características de mildiú y del cual no existe reporte alguno.

Las estructuras del patógeno, observadas en el presente trabajo de investigación, se asemejaron a la mayoría de los agentes causantes de mildiú, típicamente endoparásitos, que pertenecen al género Peronospora de la familia Peronosporaceae. Estas estructuras consistían en abundante micelio filamentoso muy ramificado y con producción de zoosporangióforos que diferencian zoosporangios apicales (Clements y Shear, 1931). Los zoosporangios observados fueron de forma elípticas, de color plomizo tal como fue descrito por Garibaldi et al. (2004) y Thines et al. (2009) en su publicación para la identificación del mildiú de la albahaca en donde reportan al género Peronospora; además, describen como nueva especie a Peronospora belbahrii.

Los resultados obtenidos en la prueba de patogenicidad fueron similares a los que obtuvieron Šafránková y Holková (2014), quienes lograron observar síntomas típicos entre los 5 a 9 días después de la inoculación; en la presente investigación, a los siete días posteriores a la inoculación se presentaron los síntomas iniciales y ligera esporulación; mientras que, a los 12 días después de la inoculación se observó que el 100 por ciento de las hojas de cada una las plantas presentaron síntomas de la enfermedad y en diferentes grados de esporulación.

Las observaciones de síntomas característicos, los datos morfométricos y los postulados de Koch, demuestran que se trata del patógeno Peronospora belbahrii el cual se halla presente en algunos lugares del Perú y debido a su acelerada tasa de desarrollo podría ser considerada como una enfermedad muy agresiva.

En la validación de métodos de evaluación de enfermedades de plantas, diversos autores han trabajado y propuesto algunas metodologías, basándose en la estimación visual y escala propuesta por Horfall y Barratt (1945). Por ejemplo Bardsley \& Ngugi (2012) midieron la exactitud y precisión del método de evaluación visual, para cuantificar la severidad de manchas foliares causado por bacterias en durazneros, en cuyo trabajo emplearon una hoja de planta como unidad muestral; del mismo modo, Nita et al. (2003) midieron la exactitud y precisión por estimación visual de mancha foliar en fresas y también consideraron como unidad muestral una hoja de la planta. En ambos casos la metodología por estimación visual fue la mejor metodología, con índices superiores a 0,80 . No obstante, debido a que el mildiú es una enfermedad que en condiciones de campo, puede ocasionar defoliación de hojas que inicia por el tercio inferior de la planta, en esta investigación se consideró como unidad muestral una planta completa, al igual que Risco y Mattos (2015) quien evaluó por el método de estimación visual directa, la severidad del mildiú de la quinua en condiciones de campo. Por este motivo, se considera la defoliación de las plantas como un factor de error para la precisión (índice que mide la repetibilidad) en evaluaciones mediante hojas en condiciones de campo (Resultados no publicados). En los trabajos previos, se obtuvieron índices superiores a 0,80 posiblemente debido a que la unidad muestral fue una hoja, donde la posibilidad de error es menor, por ese motivo, para reducir el error a causa de la defoliación, en este trabajo de investigación se empleó como unidad muestral una planta completa simulando las condiciones de campo. Por lo tanto, la elección de la mejor metodología fue basado en el valor más alto del coeficiente de correlación $\mathrm{y}$ determinación (CCC), en tal sentido, el valor promedios del CCC para el método $\mathrm{TH}$ fue 0,24; para el método HB fue de 0,49 y para el método de estimación visual directa de planta completa (VD) fue 0,55. Estos resultados evidencian que hay diferencias en exactitud y precisión en las diferentes metodologías de evaluación probadas. Por ellos, se eligió al método VD como la más exacta y la más precisa con respecto a las metodologías $\mathrm{TH}$ y $\mathrm{HB}$.

\section{Conclusiones}

De acuerdo a las observaciones de síntomas en campo, datos morfométricos y la prueba de patogenicidad, el agente causal del mildiú de la albahaca en la zona de estudio corresponde a Peronospora belbahrii.

De acuerdo al valor más alto de coeficiente de correlación $(0,55)$, la metodología de estimación visual directa de la planta completa (VD) resultó ser el mejor método de evaluación del mildiú de la albahaca.

\section{Literatura citada}

Bardsley, S. J. and Ngugi, H. K. 2012. Reliability and accuracy of visual methods to quantify severity of foliar bacterial spot symptoms on peach and nectarine. 
Plant Pathol. 62: 460-474.

Belbahri, L.; Calmin, G.; Pawlowski, J. and Lefort, F. 2005. Phylogenetic analysis and real time PCR detection of a presumably undescribed Peronospora species on sweet basil and sage. Mycol Res. 109:1276-1287.

Choi, Y. J.; Choi, I.; Lee, K. J. and Shin, H. D. 2016. First Report of Downy Mildew Caused by Peronospora balbahrii on Sweet Basil (Ocimum basilicum) in Korea. Plant Dis. 100: 2335.

Clements, F. E. and Shear, C. L. 1931. The genera of Fungi. Carnegie state Institution of Washington and United State Department of Agriculture. New York and London: Hafner Publishing Company.

Cohen, Y.; Vaknin, M.; Ben-Naim, Y.; Rubin, A. E. and Galperin, M. 2013. First Report of the Occurrence and Resistance to Mefenoxam of Peronospora belbahrii, Causal Agent of Downy Mildew of Basil (Ocimum basilicum) in Israel. Plant Dis. 97:692.

Collina, M.; Merighi, M.; Turan, C.; Pirondi, A.; Minuto, G. and Brunelli, A. 2016. First Report of Resistance of Peronospora belbahrii, causal Agent of Downy Mildew of Basil, to Mefenoxam in Italy. Plant Dis. 100:1787.

Corda, A.C.J. 1837. Icones fungorum hucusque cognitorum. 1:1-32.

Danielsen, S. y Ames, T. 2008. El mildiú de la quinua en la zona andina (Peronospora farinosa). Manual práctico para el estudio de la enfermedad y del patógeno.

Garibaldi, A.; Minuto, G.; Bertetti, D. and Gullino, M.L. 2004. Seed transmission of Peronospora sp. of basil. Journal of Plant Diseases and Protection 111, 465-469.

Homa, K.; Barney, W. P.; Ward, D. L.; Wyenandt, C. A. and Simon, J. E. 2014. Evaluation of fungicides for the control of Peronospora belbahrii on sweet basil in New Jersey. Plant Dis. 98:1561-1566.

Horsfall J. G. and Barratt, R. W. 1945. An improved grading system for measuring plant disease. (Abstr.) Phytopathology 35:655.

INEI [Instituto Nacional de Estadística e Informática]. 2015. Compendio estadístico Perú 2015. Recuperado de: https://www.inei.gob.pe/media/MenuRecursivo/ publicaciones_digitales/Est/Lib1253/cap12/cap12.pdf.

Kong, X. Y.; Wang, S.; Wan, S. L.; Xiao, C. L.; Luo, F. and Liu, Y. 2015. First Report of Downy Mildew on Basil (Ocimum basilicum) in China. Plant Dis. 99: 1642.

Lin, L. I. K. 1989. A concordance correlation coefficient to evaluate reproducibility. Biometrics 45: 255-268.

Makri, O. and Kintzios, S. 2008. Ocimum sp. (Basil): botany, cultivation, pharmaceutical properties, and biotechnology. J. Herbs Spic. Medicinal Plants 13:123150 .

Nita, M.; Ellis, M. A. and Madden, L. V. 2003. Reliability and accuracy of visual estimation of Phomopsis leaf blight of strawberry. Phytopathology 93:995-1005.
Petrželová, I.; Kitner, M.; Doležalová, I.; Ondřej, V. and Lebeda, A. 2015. First Report of Basil Downy Mildew Caused by Peronospora belbahrii in the Czech. Republic. Plant Dis. 99: 418.

Rivera, Y.; Salgado-Salazar, C.; Windham, A. S. and Crouch, J. A. 2016. Downy Mildew on Coleus (Plectranthus scutellarioides) caused by Peronospora belbahrii sensulato in Tennessee. Plant Dis. 100: 655.

Risco, M.A. y Mattos, L.L. 2015. Severidad de Peronospora variabilis en Chenopodium quinoa "Pakasankalla" como respuesta a aplicaciones de fungicidas sistémicos y bioestimulantes. Anales Cientificos 76(2) 382-398.

Šafránková, I. and Holková, L. 2014. The First Report of Downy Mildew Caused by Peronospora belbahrii on Sweet Basil in Greenhouses in the Czech Republic. Plant Dis. 98: 1579.

Saude, C.; Westerveld, S.; Filotas, M., and McDonald, M. R. 2013. First Report of Downy Mildew Caused by Peronospora belbahrii on Basil (Ocimum spp.) in Ontario. Plant Dis. 97: 1248.

Thines, M.; Telle, S.; Ploch, S. and Runge, F. 2009. Identity of the downy mildew pathogens on basil, coleus, and sage with implications for quarantine measures. Mycological research II3 532 -540. 